\title{
Should Reproductive Anatomy be Taught in University Health Courses?
}

\author{
Brent Powell ${ }^{*}$ J. Sue Fletcher \\ College of Education, California State University Stanislaus, Turlock, 95382, California, United States \\ *Corresponding Author: bpowell1@csustan.edu
}

Copyright $@ 2013$ Horizon Research Publishing All rights reserved.

\begin{abstract}
There has been little research on undergraduate reproductive anatomy education. This pilot study explores knowledge of anatomical reproductive anatomy among university students in a lower division and upper division health course. Using a Qualtrics survey program, a convenience sample of 120 lower division and 157 upper division students for a total population of 227 from one California public university were given a demographic survey prior to testing knowledge of female and male reproductive anatomy. A prediction equation validated the null hypothesis. Survey results supported the potential differentiation in what is taught in the lower and upper division classes and indicated the need for further research to assist the professor in determining the depth and breadth of information to be included in curriculum.
\end{abstract}

Keywords Health Education Content Standards, Reproductive Anatomy, Prediction Model, University Course Curriculum

\section{Introduction}

Most college/university courses and textbooks review female and male anatomical reproductive anatomy. Depending on the state, students have been taught this information in middle school, high school, and community college health courses. The Health Education Content Standards for California Public Schools: Kindergarten Through Grade Twelve was last adopted by the California State Board of Education (CSBOE) March, 2008. The health education content standards document for California includes a letter from the President of the CSBOE and State Superintendent of Public Instruction in California. The letter summarizes the importance of health education in curriculum and states the purpose of the content standards: "...provides guidance on the essential skills and knowledge that students should have at each grade level" [1].

Utilizing the health education content standards adopted in California, the established content area of Growth, Development, and Sexual Health, one of six content areas, pertains to knowledge associated with reproductive knowledge. This content area is taught only in grade categories identified as grades 5, 7 and 8, and 9 through 12 . Information on reproductive anatomy is within the first content standard labeled Essential Health Concepts. In grade 5, essential concepts students should understand as a foundation for reproductive anatomy knowledge include: "Describing the human reproductive cycle of reproduction, birth, growth, aging, and death" as well as "Explain the structure, function, and major parts of the human reproductive system" [1]. In grades 7 and 8 students should "Summarize the human reproductive cycle" and in grades 9 through 12 students should "Explain how conception occurs, the stages of pregnancy, and the responsibilities of parenting" [1].Reproductive anatomy is mentioned also in the Science Content Standards for California Public Schools: Kindergarten Through Grade Twelve, last adopted October, 1998. Only in grade 7 within the content area of Structure and Function in Living Systems does it mention "Students know how reproductive organs of the human female and male generate eggs and sperm and how sexual activity may lead to fertilization and pregnancy" [2].Although the content standards do not directly state knowledge of reproductive anatomy, anatomy knowledge is required to understand reproductive cycle, system, and conception.

Therefore, students educated within the California public school systems should have been introduced to anatomical reproductive anatomy vocabulary. Additionally, many may have been taught appropriate anatomical reproductive terms by their parents. Research pertaining to reproductive anatomy knowledge of college students is limited, thus understanding parental involvement in reproductive anatomy knowledge is primarily found within sexuality education topics. Research has been published linking beliefs by parents, students, and educators to shared responsibility in educating students on sexuality, sexual health, and reproduction [3]. However parents and students are not satisfied with communication between the parties concerning such topics [4, 5]. It has been reported that mothers are more involved with their children's education of sexual health than fathers $[6,7]$. Thus one may hypothesize mothers are more involved in reproductive anatomy 
education. Additionally, research has concluded parents' educational level and age have influence on their willingness and involvement in sexual health education. The higher the education level as well as the younger in age, parents are more involved [8]. Although mothers tend to be more involved, research finds reservations based on knowledge and comfort level of the parent. Mothers who have had formal sexual health education tend to correlate with higher knowledge and comfort levels [9]. The involvement of parental education also results from the involvement of the parent's parents, resulting in general terms and topics [10].

With these considerations college/university health professors need to determine whether to use valuable class time for re-teaching information that in theory should have been previously learned. Very little research has been published concerning undergraduate reproductive anatomy education. Knowledge of anatomy in general has been explored related to graduate programs within the medical field. For example, medical student knowledge of anatomy education has been studied, exploring their undergraduate education on the subject matter of anatomy. The purpose of the graduate research was to develop curriculum to meet the need of the medical students. Again, the research topic was specific in terms of reproductive anatomy but is a part of general anatomy. Results from the medical student study concluded that at the undergraduate level knowledge and application assessment, as well as a support process for learning anatomy were needed for success in retaining anatomy knowledge. Finally anatomy should not be limited to lower division classes in undergraduate programs, but should be taught in upper division courses as well [11].

If college/university health professors choose to reteach this information what should be included in the curriculum? This pilot study serves to initiate the conversation concerning the knowledge of reproductive anatomy in a lower division general education health course and an upper division general education health course as well as serve as a needs assessment for potential curriculum revision in those courses.

\section{Objectives}

\subsection{Research Purpose}

The purpose of this research was to statistically identify determinants of demographics as it pertained to knowledge of reproductive anatomy. By creating a prediction model of variables, we can statistically predict influence on reproductive anatomy knowledge and thus create curriculums that pertain to reproductive anatomy. The research questions of this study included:

1. Do students identify $70 \%$ of anatomical reproductive anatomy correctly?

2. What variables statistically impact student knowledge?

\subsection{Research Hypothesis}

Although the primary researchers understand the study is a pilot, the information gathered should provide a beginning for future research. The researchers hypothesized results based on the two research questions. Because students in both courses should have been exposed to reproductive anatomy in their pre-college health/science courses and parents, students in the upper division course theoretically have completed their lower division general health course. This additional knowledge should provide increased knowledge related to the subject matter. Therefore, researchers hypothesized:

1. Upper division students are significantly more knowledgeable statistically of anatomical reproductive anatomy.

Additionally, because of the many demographical variables assessed in the study, researchers hypothesized:

2. There is a significant difference statistically between variables' impact on student knowledge of anatomical reproductive anatomy.

\section{Methods}

\subsection{Research Design}

This study utilized a non-probability, cross-sectional research design because no random sampling was conducted and data was collected from research participants at a single point in time [12]. The research survey was designed to gather demographic information as well as knowledge related to correctly identifying ten female and ten male anatomical reproductive parts. The survey was administered using an online software system for survey programs called Qualtrics, with a link to the survey delivered to the research participants. Qualtrics, like other online survey tools, is used for generating the survey, and collecting results from the administered survey.

The University Institutional Review Board approved the research study with informed consent obtained via the online system used to develop and administer the survey using the Qualtrics survey software system. Permission was granted to conduct the study when participants check the "yes" box giving their consent to participate before the web-based survey will continue. Consent instruction was self-administered during the study. Consent or permission was provided by the individuals who are of age 18 years and older.

To avoid possible coercion or undue influence, the survey was voluntary and in no way was language for participation coercive. English was the language prospective participants utilized for granting consent.

\subsection{Inclusion/Exclusion Criteria and Sample}

Inclusion to the study was limited to students at California State University Stanislaus enrolled in KINS 1000-Health in 
Today's Society and KINS 4330-Family Health. This was a population of students representing those students who will be learning anatomical reproductive anatomy in addition to other health education topics. Only students age 18 and older were included in the study. Recruitment of participants was conducted during the meeting times of each course. Participants were mainly students from the primary investigators' KINS 1000 and KINS 4330 courses. Recruitment of participants came from KINS 1000 and KINS 4330 course registration. It was up to the instructor if he or she wishes to grant extra credit for participation.

The sample surveyed in this pilot study included 120 students in a lower division course KINS 1000-Health in Today's Society and 157 students in an upper course KINS 4330-Family Health, making the total population 227. Incomplete surveys were not to be included in the study.

\subsection{Research Duration}

Students enrolled in the KINS 1000 and KINS 4330 courses were delivered the link via email and/or Blackboard prior to reproductive anatomy being taught in the courses. Each course instructor was consulted and the appropriate survey administration time was determined. Students were given two weeks to complete the survey. A reminder was sent out to students after one week.

\subsection{Analysis}

The data were extracted from Qualtrics and imported in the Statistical Package for the Social Sciences (SPSS).
Analysis of data included frequency tables of demographic variables as well as reproductive anatomy identification questions. The reproductive anatomy questions were coded to represent correct and incorrect answers. A multiple linear regression predication analysis was calculated to examine the extent to which the independent variables (demographic variables) predict the dependent variable (reproductive anatomy identification). The resulting frequency tables and multiple linear regression prediction equation were used to address the research questions. All independent variables were entered at one time and then variables were removed one at a time based on a preset significance value, in this case $p<.05$. The regression equation was validated with a subset of data reserved for validation purposes.

\section{Results}

There was $33 \%$ response rate or 79 students to the survey. The independent variables tested were: instructor/course; age; cultural identification; gender; high school attended; student marital status; sexual orientation; parent's marital status; student born in US; parents born in US; grandparents born in US; primary source of reproductive anatomy education; secondary source of reproductive anatomy education; school based sex education; primary vocabulary concerning reproductive anatomy; and age became aware of reproductive anatomy. The largest response for each demographical question is represented in Table 1 as percentage.

Table 1. Demographic variable frequencies

\begin{tabular}{|c|c|c|}
\hline Question & Largest Response & Percent \\
\hline Gender & Female & 49.5 \\
\hline Age & $20-21$ & 40.5 \\
\hline Cultural Group & Mexican American & 82.3 \\
\hline High School & CSUS Area & 86.1 \\
\hline Marital Status (self) & Single & 69.6 \\
\hline Marital Status (parents) & Married & 88.6 \\
\hline Sexual Orientation & Heterosexual & 84.8 \\
\hline Born in US (self) & Yes & $50.6 / 53.2$ \\
\hline Born in US (Mother/Father) & Yes / No & $60.8 / 70.9$ \\
\hline Born in US (Grandmother/father) & No / No & $46.8 / 31.6$ \\
\hline Primary source of reproductive anatomy & School / Friends & $24.1 / 22.8$ \\
\hline Secondary source of reproductive anatomy & School / Friends & 35.4 \\
\hline Educational levels sex education taught & Elem. & 60.8 \\
\hline (\% for yes) & Middle & 62.0 \\
\hline Primary vocabulary use for reproductive anatomy & JR-College & 21.5 \\
\hline
\end{tabular}


Table 2. Knowledge of reproductive anatomy

\begin{tabular}{|c|c|c|c|}
\hline Female Anatomy & $\begin{array}{c}\text { Total \% Correct } \\
\text { (Male vs Female) }\end{array}$ & Male Anatomy & $\begin{array}{l}\text { Total \% Correct } \\
\text { (Male vs Female) }\end{array}$ \\
\hline Ovary & $\begin{array}{c}91.1 \\
(90.6 / 91.5)\end{array}$ & Vas Deferens & $\begin{array}{c}34.2 \\
(47.1 / 21.3)\end{array}$ \\
\hline Fallopian Tube & $\begin{array}{c}88.6 \\
(87.5 / 89.7) \\
\end{array}$ & Urethra & $\begin{array}{c}73.4 \\
(78.6 / 68.1) \\
\end{array}$ \\
\hline Uterus & $\begin{array}{c}88.6 \\
(93.8 / 83.4)\end{array}$ & Penis & $\begin{array}{c}74.7 \\
(77.0 / 72.3)\end{array}$ \\
\hline Cervix & $\begin{array}{c}82.3 \\
(78.1 / 86.5)\end{array}$ & Epididymis & $\begin{array}{c}46.8 \\
(59.4 / 34)\end{array}$ \\
\hline Vagina or Vaginal Canal & $\begin{array}{c}92.4 \\
(87.5 / 97.2)\end{array}$ & Urethra Opening & $\begin{array}{c}63.3 \\
(60.6 / 66.0)\end{array}$ \\
\hline Clitoris & $\begin{array}{c}84.8 \\
(84.4 / 85.1)\end{array}$ & Testicle & $\begin{array}{c}86.1 \\
(84.9 / 87.2)\end{array}$ \\
\hline Urethra & $\begin{array}{c}67.1 \\
(71.9 / 62.2)\end{array}$ & Scrotum & $\begin{array}{c}88.6 \\
(87.6 / 89.4)\end{array}$ \\
\hline Vulva & $\begin{array}{c}34.2 \\
(28.1 / 40.3)\end{array}$ & Cowper's Gland & $\begin{array}{c}24.1 \\
(31.3 / 17)\end{array}$ \\
\hline Vaginal Opening & $\begin{array}{c}32.9 \\
(31.3 / 34.5)\end{array}$ & Prostate Gland & $\begin{array}{c}59.5 \\
(70.0 / 48.9)\end{array}$ \\
\hline Anus or Rectum & $\begin{array}{c}96.2 \\
(96.9 / 95.7)\end{array}$ & Anus or Rectum & $\begin{array}{c}92.4 \\
(90.9 / 93.6)\end{array}$ \\
\hline
\end{tabular}

Table 3. KINS 1000 Knowledge of reproductive anatomy

\begin{tabular}{|c|c|c|c|}
\hline Female Anatomy & Total \% Correct & Male Anatomy & Total \% Correct \\
\hline Ovary & 79.0 & Vas Deferens & 65.8 \\
\hline Fallopian Tube & 85.4 & Urethra & 74.3 \\
\hline Uterus & 83.6 & Penis & 53.2 \\
\hline Cervix & 76.3 & Epididymis & 84.6 \\
\hline Vagina or Vaginal Canal & 86.4 & Trethra Opening & 82.6 \\
\hline Clitoris & 84.4 & Scrotum & 15.7 \\
\hline Urethra & 71.5 & Cowper's Gland & 50.6 \\
\hline Vulva & 36.7 & Prostate Gland & 88.1 \\
\hline Vaginal Opening & 39.4 & Anus or Rectum & \\
\hline Anus or Rectum & 100 & & \\
\hline
\end{tabular}

Table 4. KINS 4330 knowledge of reproductive anatomy

\begin{tabular}{|c|c|c|c|}
\hline Female Anatomy & Total \% Correct & Male Anatomy & Total \% Correct \\
\hline Ovary & 96.2 & Vas Deferens & 73.5 \\
\hline Fallopian Tube & 93.1 & Urethra & 72.5 \\
\hline Uterus & 88.5 & Penis & 47.0 \\
\hline Cervix & 83.8 & Epididymis & 69.3 \\
\hline Vagina or Vaginal Canal & 93.9 & Trethra Opening & 86.2 \\
\hline Clitoris & 86.1 & Scrotum & 26.2 \\
\hline Urethra & 66.9 & Cowper's Gland & 61.5 \\
\hline Vulva & 35.4 & Prostate Gland & 93.8 \\
\hline Vaginal Opening & 32.3 & Anus or Rectum & \\
\hline Anus or Rectum & 93.9 & & \\
\hline
\end{tabular}


Table 5. Significant independent variables

\begin{tabular}{|c|c|}
\hline Independent Variable & Significance \\
\hline Culture & $\mathrm{p}=.051$ \\
\hline Gender & $\mathrm{p}=.013$ \\
\hline Student born in US & $\mathrm{p}=.025$ \\
\hline Mother born in US & $\mathrm{p}=.004$ \\
\hline Primary source of reproductive anatomy education & $\mathrm{p}=.001$ \\
\hline Secondary source of reproductive anatomy education & $\mathrm{p}=.005$ \\
\hline
\end{tabular}

The dependent variable of reproductive anatomy consisted of identification of ten female reproductive anatomy parts: Ovary; fallopian tube; uterus; cervix; vaginal canal; clitoris; urethra; vulva; vaginal opening; and anus/rectum. In addition identification of ten male reproductive anatomy parts were assessed: Vas deferens; urethra; penis; epididymis; urethra opining; testicle; scrotum; Cowper's gland; prostate gland; and anus/rectum. Identification of reproductive anatomy utilized a cross-sectional diagram depicting the female and male anatomy. Each anatomic reproductive area was labeled with a number. Students were not provided any information related to anatomical terms and were asked to identify the ten female and ten male reproductive parts. Table 2 presents knowledge of female and male reproductive anatomy by correct percentage for all respondents.

When analyzing the courses in isolation of one another, KINS 1000 responses consisted of $39 \%(n=31)$ of total responses. Additionally, KINS 1000 respondents correctly identified $74.3 \%$ of female reproductive anatomy and $58.7 \%$ of male reproductive anatomy; thus correctly identifying $66.5 \%$ of anatomical reproductive anatomy. Table 3 presents knowledge of female and male reproductive anatomy by correct percentage for KINS 1000 respondents.

KINS 4330 represented $61 \%(n=48)$ of total respondents. Respondents in KINS 4330 correctly identified $77 \%$ of female anatomy and $66 \%$ of male anatomy therefore correctly identifying $71.5 \%$ of anatomical reproductive anatomy. Table 4 presents knowledge of female and male reproductive anatomy by correct percentage for KINS 4330 respondents.

Due to a smaller sample size a more lenient significance level was chosen. Of the demographic variables some were found to be significant at the .05 level in predicting reproductive anatomy score: Culture; gender; if participant was born in the US; if participant's mother was born in the US; primary source of reproductive anatomy; and primary vocabulary used for reproductive anatomy. Culture was .051 but was left in by the researchers due to its proximity to the .05 level. Table 5 presents the independent variables found to be significant.

A prediction equation was developed to test null hypotheses; there is no difference between predicted knowledge score and actual knowledge scores.

Knowledge Score of Reproductive Anatomy $=19.315$
+.180 (culture) +1.650 (gender) -2.259 (self-born in US) + 3.676 (mother born in US) - .531 (source of reproductive anatomy) +1.609 (primary vocabulary)

The equation was validated testing null hypothesis: There is no difference between actual knowledge score of reproductive anatomy and predictive knowledge score of reproductive anatomy. The null hypothesis was accepted; there was no difference, with high correlation found between the predictive score and actual score. Since the two variables should represent the same score, a correlation was found to be .548. Very little observed difference between actual reproductive anatomy score and predictive score was found within the mean of the paired sample test. Table 6 presents a paired samples test.

Table 6. Paired samples test

\begin{tabular}{|c|c|c|}
\hline & $\mathrm{t}$ & Sig. (2-tailed) \\
\hline total score -predscore & .046 & .964 \\
\hline
\end{tabular}

The observed difference between means of actual score and predictive score was $4.6 \%$ the size of the difference that would be expected due to random sample fluctuation alone. And the significance is more than .01 therefore we accept the null hypothesis. Difference of that magnitude observed would not occur due to random sample fluctuation alone since the means are equal $96.4 \%$ of the time.

\section{Discussion}

This study was guided by two research questions. The first research question asked do students identify $70 \%$ of anatomical reproductive anatomy correctly. The total percent correct for both male and female reproductive anatomy was $70.1 \%$. Therefore students in KINS 1000 and KINS 4330 do identify $70 \%$ of reproductive anatomy correctly. As previously presented there are significant differences between the knowledge of male versus females as well as between courses. The researchers were correct in hypothesizing KINS 4330 students would score higher than the lower division course of KINS 1000; however the difference was only $5 \%$ and thus was not statistically significant.

The second research question, what variables statistically 
predict reproductive anatomy knowledge, requires an understanding of the significant predictors in the equation.

Knowledge Score of Reproductive Anatomy $=19.315$ +.180 (culture) +1.650 (gender) -2.259 (self-born in US) + 3.676 (mother born in US) - .531 (source of reproductive anatomy) +1.609 (primary vocabulary)

Culture has a small positive relationship to the dependent variable; knowledge score of reproductive anatomy. Students who were Mexican American were more likely to know reproductive anatomy. If there were fewer choices, the primary investigators believe there would be a stronger relationship. Students born in the US had a high negative relationship to the dependent variable. Thus those born in the US were less likely to know reproductive anatomy. The third significant variable, if the student's mother was born in the US had a high positive relationship with the dependent variable. This finding suggests that students whose mother was born in the US were more likely to know reproductive anatomy. This is somewhat consistent with reports that mothers are more involved with their children's education of sexual health than fathers are [6, 7]. Additionally this supports previously discussed hypothesis of mothers being more involved in reproductive anatomy education as well. The source of the student's reproductive anatomy knowledge has a small positive relationship with the dependent variable. Those who learned reproductive anatomy from a person rather than religion, culture, or bible were more likely to know reproductive anatomy. Finally, primary vocabulary has a positive relationship, thus those who use anatomical names instead of slang terminology were more likely to correctly identify reproductive anatomy.

\section{Conclusions}

This needs assessment survey using a non-probability cross-sectional research design with no random sampling resulted in identifying potential differentiation in what is taught in lower and upper division university courses in regard to anatomic reproductive anatomy. Results from the online Qualtrics survey program using a prediction equation validated the null hypothesis. Further research on the knowledge score of reproductive anatomy and predictive variables need to be assessed in future research using comparisons with pre- and post-instruction by all instructors to determine the significance of instruction style. An assessment of knowledge at the beginning of the course would assist the professor in determining the depth and breadth of information to be included in the curriculum.

Although this pilot was limited to one university, the aim was to begin to inform instructors of areas of curriculum development. Because research on the topic of higher education curriculum and student knowledge of reproductive anatomy is limited, this study was designed as a pilot study to identify areas in which to inform research on a larger, longitudinal study.

These results should be interpreted as a pilot study.
Limitations of the study reflect the small response rate. Additionally those students who did not complete the survey may have less knowledge of reproductive anatomy and/or lower comfort levels with the topic. Modifications to the survey are needed resulting from the pilot study. For example, the small relationship between culture and the dependent variable is hypothesized to be due to the many cultural choices $(20+$ choices) presented on the survey. Furthermore, the small positive relationship between reproductive anatomy and the source of student knowledge may also yield better results if fewer choices are provided within the survey. The survey did not include questions related to attitude of reproductive anatomy knowledge, or the perception of their reproductive anatomy education from both a parental and public education point of view. To add to the understanding of prior educational experience, future research should assess a public versus private education as a variable and possible correlation to reproductive anatomy knowledge.

\section{REFERENCES}

[1] California State Board of Education. (2008). Health Education Content Standards for California Public Schools. Online available from http://www.cde.ca.gov/be/st/ss/docum ents/healthstandmar08.pdf

[2] California State Board of Education. (1998). Science Content Standards for California Public Schools. Online available from

http:/www.cde.ca.gov/be/st/ss/documents/sciencestnd.pdf

[3] Croft, C.A. \& Asmussen, L. (1992). Perceptions of mothers, youth, and educators: A path toward détente regarding sexuality education. Family Relations, 41, 452-459.

[4] Feldman, S.S., \& Rosenthal, D.A. (2000). The effect of communication characteristics on family members' perceptions of parents as sex educators. Journal of Research on Adolescence, 10, 119-129.

[5] Weaver, A.D., Byers, E.S., Sears, H.A., Cohen, J. N., \& Randall, H. E. S. (2002). Sexual health education at school and at home: Attitudes and experiences of New Brunswick parents. Canadian Journal of Human Sexuality, 11, 20-24.

[6] Fisher, T.D. (1990). Characteristics of mothers and fathers who talk to their adolescent children about sexuality. Journal of Psychology \& Human sexuality, 3(2), 62-64.

[7] Meschke, L. L., Bartholomae, S., \& Zentall, S. R. (2002). Adolescent sexuality and parent-adolescent process: Promoting healthy teen choices. Journal of Adolescent Health, 31, 264-269.

[8] Reddy, K. S. (1994). Parents', teachers', and students' attitudes toward sex education. Journal of Psychological Researches, 28, 176-177.

[9] Jaccard, J., Dodge, T., \& Dittus, P. (2002). Parent-adolescent communication about sex and birth control: A conceptual framework. In S.S. Feldman \& D. A. Rosenthal (Eds.), Talking sexuality: Parent-adolescent communication (pp. 
9-41). San Francisco, CA: Jossey-Bass.

[10] Byers, S. E., Sears, H. A., \& Weaver, A. D. (2008). Parents' reports of sexual communication with children in kindergarten to grade 8. Journal of Marriage and Family 70, 92-95.

[11] Smith, C. F. \& Mathias, H. S. (2011). What impact does anatomy education have on clinical practice? Journal of Clinical Anatomy 24. Doi: 10.1002/ca.21065

[12] Gay, L.R., Mills, G.E., \& Airasian, P. (2009). Educational research: Competencies for analysis and applications ( $\left.{ }^{9 \text { th }} \mathrm{ed}\right)$. Upper Saddle River, NJ: Pearson.
[13] Mertler, C., \& Vannatta, R. (2005). Advanced and multivariate statistical methods. (3rd ed). Glendale, CA: Pyrczak Publishing.

[14] Stevens, J. (1992). Applied multivariate statistics for the social sciences (2nd ed). Hillsdale, NH: Lawrence Erlbaum Associates.

[15] Tabachnick, B.G. \& Fidell, L.S. (1996). Using multivariate statistics $\left({ }^{\text {3rd }}\right.$. ed). New York: HarperCollins 